\title{
84 Krafttraining
}

(C) Springer-Verlag GmbH Deutschland, ein Teil von Springer Nature 2018

D. Mathias, Fit und gesund von 1 bis Hundert

https://doi.org/10.1007/978-3-662-56307-6_84

Ausdauer, mehr aber noch Kraftsport führen zu Muskelwachstum und Kraftzuwachs. Krafttraining

- stabilisiert Knochen und Gelenke,

- verringert das Osteoporoserisiko,

- steigert den Grundumsatz mit der Folge von Gewichtsabnahme und Optimierung des Stoffwechsels.

Die Stärke der Skelettmuskulatur erreicht bei Frauen ungefähr mit dem 21. und bei Männern mit dem 25. Lebensjahr ihr individuelles Maximum. Die 656 Muskeln machen dann bei Frauen etwa 35 \% des Körpergewichts aus, bei Männern sind es ca. $40 \%$. Altersbedingt vermindert sich diese Muskelmasse aber wieder, vom 30. bis zum 55. Lebensjahr langsam und danach im beschleunigten Maße. Davon sind in der Regel die unteren Extremitäten mehr betroffen. Der Gesamtverlust an Muskulatur kann dann bis zum Alter von 75 Jahren 25-35 \% betragen. Neben Sport verlangsamt ein vermehrter Proteinanteil in der Nahrung von etwa 1,2 g/kg Körpergewicht diesen Abbauprozess ( $\triangleright$ Kap. 5).

\section{Der Abbau von Muskulatur bewirkt eine ungünstige Verschiebung des Kraft-Last-Ver- hältnisses.}

Die Entlastung von Gelenken, Bändern und Sehnen durch starke Muskeln ist nicht mehr gegeben. Das Missverhältnis von Belastung und Belastbarkeit erhöht die Gefahr von Stürzen und Verletzungen, Einschränkungen in den Alltagsaktivitäten werden so zum Dauerproblem. Mit regelmäßigem und abwechslungsreichem Training lässt sich jedoch die Kraft und auch die Knochenstärke der jungen Jahre

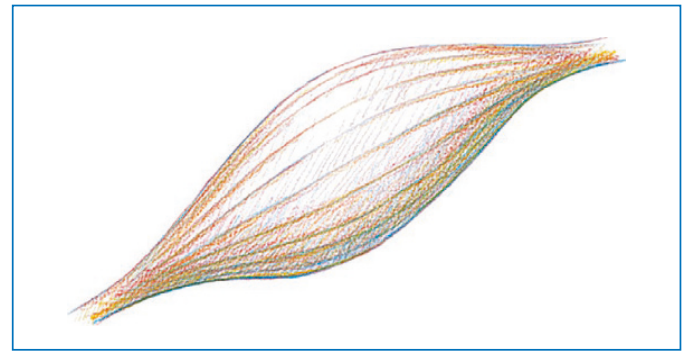

- Abb. 84.1

nahezu erhalten. Die dabei geforderten über hundert großen Skelettmuskeln sind bis ins hohe Alter trainierbar. Beispiele für kleine Skelettmuskeln wären die 17 Lachmuskeln und die 40 Gesichtsmuskeln für das Stirnrunzeln.

Muskeln sind aber nicht nur Arbeitsmaschinen. Das wird deutlich bei Betrachtung der Faserzusammensetzung der Muskelnerven. Nicht einmal $20 \%$ der Nervenfasern sind für motorische Aufgaben bestimmt. Gut $80 \%$ der Fasern bestehen dagegen etwa hälftig aus vasomotorischen und aus sensorischen Anteilen (Leyk 2009). Die vasomotorischen Fasern sorgen schon bei kurzer Beanspruchung für eine sofortige Steigerung der lokalen Durchblutung im Muskel und tragen damit zur Stärkung des Herz-Kreislauf-Systems bei. Sehr ausgeklügelt reagieren die sensorischen Fasern des Muskelnervs. Sie beeinflussen über eine komplexe Vernetzung mit dem Zentralnervensystem wichtige Regelsysteme im Körper. Und die Muskeln produzieren eine Fülle von wichtigen Botenstoffen, die entscheidend in die Stoffwechselprozesse eingreifen ( Kap. 54). Die positiven Effekte von Sport auf Psyche oder auf das Immunsystem finden so eine zusätzliche Erklärung. 